\title{
Genetics and adverse events with irinotecan treatment: what do we know?
}

\author{
David Páez ${ }^{* 1,2}$ \\ ${ }^{1}$ Medical Oncology Department, Hospital de la Santa Creu i Sant Pau, Barcelona, Spain \\ ${ }^{2}$ CIBERER U-705, Barcelona, Spain \\ *Author for correspondence: Tel.: +34 93 5565638; Fax: +34 93 5565769; dpaez@santpau.cat
}
"the clinical utility of routine UGT1A1 genotyping to pre-emptively adjust irinotecan dosage reduces toxicity while maintaining drug efficacy"

First draft submitted: 5 February 2019; Accepted for publication: 3 April 2019; Published online: 23 May 2019

The topoisomerase I inhibitor irinotecan is a cytotoxic drug that is commonly used in the treatment of advanced solid tumors such as colorectal cancer. It is usually co-administered with infusional 5-fluorouracil/leucovorin (FOLFIRI regimen), and when combined with targeted therapies such as bevacizumab or cetuximab it is a standard first-line option for patients with metastatic colorectal cancer $[1,2]$. Treatment with irinotecan has an acceptable tolerance profile in most patients, although as many as $20-35 \%$ of patients develop severe adverse toxicities, including delayed-type diarrhea and myelosuppresion [3,4]. Such toxicities may occur due to a deficiency of enzymes involved in the metabolism of the drug.

The metabolic conversion of irinotecan to the active metabolite SN-38 is mediated by carboxylesterase enzymes. $\mathrm{SN}-38$ is subsequently conjugated predominantly by the enzyme UGT1A1. In the promoter region of the UGT1A1 gene, an extra TA dinucleotide results in the $U G T 1 A 1^{*} 28$ variant allele. Patients harboring an homozygous genotype for this allele $\left(U G T 1 A 1^{*} 28 /{ }^{*} 28\right)$, also known as Gilbert's syndrome, have a reduction in the enzyme activity by up to $70 \%$, and are more likely to develop severe irinotecan-induced toxicity [5-8]. As premature dose reduction and administration delays due to toxicity may decrease antitumor activity, it would be of interest to anticipate serious toxicity rates that decrease survival by genetic analysis prior to treatment, thereby optimizing and personalizing irinotecan dosing. Although in routine clinical genotyping is not yet fully established, available evidence suggests that patient safety and clinical outcomes could be substantially improved by upfront UGT1A1 screening and UGT1A1guided dose individualization. For this reason, in 2004, the term irinogenetics was coined as a promising model for the application of pharmacogenetics to personalize this drug therapy [9]. The final goal was to predict, diminish and/or avoid toxicity and to improve the therapeutic effect of irinotecan. Since then, numerous pharmacogenetic studies have explored single-nucleotide polymorphisms and haplotypes in UGT1A1 and other genes involved in the metabolism and excretion of this drug and its metabolites.

In 2005, the US FDA recognized the importance of the pharmacogenetics of irinotecan and added a warning in the summary of product characteristics stating that patients with the $U G T 1 A 1^{*} 28$ homozygous genotype should be given a reduced dose [10]. Despite the good intentions behind this recommendation, however, the UGT1A1 genotype is rarely used as a predictive biomarker of irinotecan toxicity in everyday practice. One reason for this is probably because in 2007, a meta-analysis of nine studies including a variety of irinotecan-containing regimens demonstrated that the $U G T 1 A 1^{*} 28$ genotype-based dosing of irinotecan is likely to improve safety of patients on high doses of irinotecan $\left(>250 \mathrm{mg} / \mathrm{m}^{2}\right)$ but not in those on low doses $\left(<150 \mathrm{mg} / \mathrm{m}^{2}\right)$ [11]. However, in 2011, a Phase I-IV dose-finding trial confirmed that in patients with the UGT1A1*28 homozygous genotype, the maximum tolerated dose was $30 \%$ lower than the standard dose of $180 \mathrm{mg} / \mathrm{m}^{2}$ [12]. It remains to be seen whether such a low dose could be less effective and, thus, other more active chemotherapeutic regimen should be considered for these patients. In 2014, a collaborative meta-analysis evaluated the relationship between the UGT1A1 genotype and patient survival following irinotecan therapy. Overall, no statistically significant differences in survival were found between patients harboring different UGT1A1 genotypes (homozygous, heterozygous or wild-type). Additionally, the irinotecan dose, the chemotherapy regimen or the treatment line of therapy did not have either an impact on survival [13]. It 
would seem, therefore, that the clinical utility of routine UGT1A1 genotyping to pre-emptively adjust irinotecan dosage reduces toxicity while maintaining drug efficacy. More recently, a retrospective cost analysis confirmed a higher predicted cost per patient for $U G T 1 A 1^{*} 28$ homozygous genotypes versus other UGT1A1 genotypes. The different toxicity profile and a higher frequency of costly interventions such as hospitalizations, represent yet another step toward the confirmation of the clinical utility of testing UGT1A1 [14].

Even though the risk of severe irinotecan-related toxicity can be significantly decreased if the initial dose in UGT1A1*28 homozygous patients is reduced, not all toxicity can be prevented with this strategy. Factors such as other $U G T 1 A 1$ variants can also influence UGT1A1 enzyme activity [15]. In the Asian population, where the $U G T 1 A 1^{*} 28$ allele frequency is less than 0.16 , a more common variant, $U G T 1 A 1^{*} 6$, can predict irinotecan-induced toxicities better than the $U G T 1 A 1^{*} 28$ polymorphism [16]. Furthermore, not all treatment-related toxicity can be explained by reduced UGT1A1 activity. Other UGT1A isoenzymes, carboxylesterases, CYP3As and adenosine triphosphate binding cassette transporters are involved in the metabolism of irinotecan and have a putative role on irinotecan-related toxicity. In line with this reasoning, patient safety could be further improved by expanding the number of tested variants considering that irinotecan is usually co-administered with other agents, mainly 5-fluorouracil. The clinical value of the DPYD genotype, the gene encoding the main metabolic enzyme for fluoropyrimidine inactivation, has been demonstrated in a prospective genotype-guided dosing study [17]. Moreover, recent findings showing upfront $D P Y D$-guided dose individualization is cost neutral which further supports the implementation of DPYD screening prior to fluoropyrimidine treatment [18].

Additionally, a genotype-driven dosing strategy could be applied to patients with a favorable genotype. Various phase I dose escalation trials have demonstrated that $U G T 1 A 1$ wild-type and heterozygous patients can tolerate significantly higher doses of irinotecan than the standard approved dose. In 2010, Toffoli et al. established the maximum tolerated doses of irinotecan in $370 \mathrm{mg} / \mathrm{m}^{2}$ and $310 \mathrm{mg} / \mathrm{m}^{2}$ for $U G T 1 A 1{ }^{*} 1 /{ }^{*} 1$ and ${ }^{*} 1 /{ }^{*} 28$ patients, respectively [19] and similar doses were safely administered in a validation study published the following year[12]. A third study, conducted in patients treated with the FOLFIRI plus bevacizumab combination, established the maximum tolerated doses of irinotecan in 310 and $260 \mathrm{mg} / \mathrm{m}^{2}$ for $U G T 1 A 1{ }^{*} 1 /{ }^{*} 1$ and ${ }^{*} 1 /{ }^{*} 28$ patients, respectively [20]. More recently, in a multicenter randomized Phase II trial in metastatic colorectal cancer patients, high doses of irinotecan according to the UGT1A1 genotype were administered. This genotype-guided dose strategy increased the objective response rate without increasing toxicity in patients with a favorable UGT1A1 genotype $\left({ }^{*} 1 /{ }^{*} 1\right.$ or ${ }^{*} 1 /{ }^{*} 28$ [21]. Continuing this line of research, several ongoing clinical trials are exploring the clinical benefit of higher doses of irinotecan from a genotype-driven dosing approach (ClinicalTrials.gov identifier NCT02138617, NCT02256800).

Despite the progress made in our understanding of irinotecan pharmacogenetics, specific dose-adjustment recommendations are pending in drug label and clinical practice guidelines for $U G T 1 A 1^{*} 28$ homozygous patients. Moreover, a well-powered randomized-controlled trial in wild-type and heterozygous patients is needed to compare standard care with irinotecan intensification according to UGT1A1 genotype status. In the era of personalized medicine it is simply a matter of time until clinicians consider implementing pre-emptive genotyping strategies to guide dosing for both 5-FU and irinotecan drugs so as to reduce toxicity and optimize their therapeutic effect.

Financial \& competing interests disclosure

The author declares a scientific advisory role for Amgen, Sanofi, Merck Serono, F Hoffmann-La Roche Ltd, Lilly, Kyowa and Servier. The authors have no other relevant affiliations or financial involvement with any organization or entity with a financial interest in or financial conflict with the subject matter or materials discussed in the manuscript apart from those disclosed.

No writing assistance was utilized in the production of this manuscript.

\section{References}

1. Saltz LB, Clarke S, Díaz-Rubio E et al. Bevacizumab in combination with oxaliplatin-based chemotherapy as first-line therapy in metastatic colorectal cancer: a randomized Phase III study. J. Clin. Oncol. 26(12), 2013-2019 (2008).

2. Van Cutsem E, Kohne CH, Hitre E et al. Cetuximab and chemotherapy as initial treatment for metastatic colorectal cancer. N. Engl. J. Med. 360(14), 1408-1417 (2009).

3. Ducreux M, Ychou M, Seitz JF et al. Irinotecan combined with bolus fluorouracil, continuous infusion fluorouracil, and high-dose leucovorin every two weeks (LV5FU2 regimen): a clinical dosefinding and pharmacokinetic study in patients with pretreated metastatic colorectal cancer. J. Clin. Oncol. 17(9), 2901-2908 (1999). 
4. Fuchs CS, Melvin RM, Harker G et al. Phase III comparison of two irinotecan dosing regimens in second-line therapy of metastatic colorectal cancer. J. Clin. Oncol. 21(5), 807-814 (2003).

5. Marcuello E, Altés A, Menoyo A et al. UGT1A1 gene variations and irinotecan treatment in patients with metastatic colorectal cancer. Br. J. Cancer 91(4), 678-682 (2004).

6. Toffoli G, Cecchin E, Corona G et al. The role of UGT1A1*28 polymorphism in the pharmacodynamics and pharmacokinetics of irinotecan in patients with metastatic colorectal cancer. J. Clin. Oncol. 24(19), 3061-3068 (2006).

7. Innocenti F, Undevia SD, Iyer L et al. Genetic variants in the UDP-glucuronosyltransferase $1 \mathrm{~A} 1$ gene predict the risk of severe neutropenia of irinotecan. J. Clin. Oncol. 22(8), 1382-1388 (2004).

8. Rouits E, Boisdron-Celle M, Dumont A et al. Relevance of different UGT1A1 polymorphisms in irinotecan-induced toxicity: a molecular and clinical study of 75 patients. Clin. Cancer Res. 10(15), 5151-5159 (2004).

9. Innocenti F, Ratain MJ. "Irinogenetics" and UGT1A: from genotypes to haplotypes. Clin. Pharmacol. Ther. 75(6), 495-500 (2004).

10. US FDA: camptosar label. www.fda.gov/cder/foi/label/2005/020571s024,027,028lbl.pdf

11. Hoskins JM, Goldberg RM, Qu P et al. UGT1A1*28 genotype and irinotecan-induced neutropenia: dose matters. J. Natl Cancer Inst. 99(17), 1290-1295 (2007).

12. Marcuello E, Páez D, Paré L et al. A genotype-directed Phase I-IV dose-finding study of irinotecan in combination with fluorouracil/leucovorin as first-line treatment in advanced colorectal cancer. Br. J. Cancer 105(1), 53-57 (2011).

13. Dias MM, Pignon JP, Karapetis CS et al. The effect of the $U G T 1 A 1{ }^{*} 28$ allele on survival after irinotecan-based chemotherapy: a collaborative meta-analysis. Pharmacogenomics J. 14(5), 424-431 (2014).

14. Roncato R, Cecchin E, Montico M et al. Cost evaluation of irinotecan-related toxicities associated with the UGT1A1*28 patient genotype. Clin. Pharmacol. Ther. 102(1), 123-130 (2017).

15. Riera P, Salazar J, Virgili AC et al. Relevance of CYP3A4*20,UGT1A1*37 and UGT1A1*28 variants in irinotecan-induced severe toxicity. Br. J. Clin. Pharmacol. 84(6), 1389-1392 (2018).

16. Han FF, Guo CL, Yu D et al. Associations between $U G T 1 A 1^{*} 6$ or $U G T 1 A 1^{*} 6 /{ }^{*} 28$ polymorphisms and irinotecan-induced neutropenia in Asian cancer patients. Cancer Chemother. Pharmacol. 73(4), 779-788 (2014).

17. Henricks LM, Lunenburg CATC, de Man FM et al. DPYD genotype-guided dose individualisation of fluoropyrimidine therapy in patients with cancer: a prospective safety analysis. Lancet Oncol. 19(11), 1459-1467 (2018).

18. Henricks LM, Lunenburg CATC, de Man FM et al. A cost analysis of upfront DPYD genotype-guided dose individualisation in fluoropyrimidine-based anticancer therapy. Eur. J. Cancer. 107, 60-67 (2019).

19. Toffoli G, Cecchin E, Gasparini G et al. Genotype-driven Phase I study of irinotecan administered in combination with fluorouracil/leucovorin in patients with metastatic colorectal cancer. J. Clin. Oncol. 28(5), 866-871 (2010).

20. Toffoli G, Sharma MR, Marangon E et al. Genotype-guided dosing study of FOLFIRI plus bevacizumab in patients with metastatic colorectal cancer. Clin. Cancer Res. 23(4), 918-924 (2017).

21. Páez D, Tobeña M, Fernández-Plana J et al. Pharmacogenetic clinical randomised Phase II trial to evaluate the efficacy and safety of FOLFIRI with high-dose irinotecan (HD-FOLFIRI) in metastatic colorectal cancer patients according to their UGT1A 1 genotype. Br. J. Cancer 120(2), 190-195 (2019). 
